\title{
The first reported case of Noonan syndrome complicated with hepatocellular carcinoma.
}

\author{
Satoru Kakizaki ${ }^{1}$, Daisuke Uehara ${ }^{2}$, Hiroki Tojima², Takayoshi Suga ${ }^{2}$, Yuichi Yamazaki², \\ Ken Sato ${ }^{2}$, Norio Kubo ${ }^{2}$, Ken Shirabe ${ }^{2}$, Takayuki Yokota ${ }^{2}$, Kei Shibuya ${ }^{2}$, Tatsurou \\ Maehara $^{2}$, Hideaki Yokoo ${ }^{2}$, Atsushi Naganuma ${ }^{1}$, and Toshio Uraoka² \\ ${ }^{1}$ National Hospital Organization Takasaki General Medical Center \\ ${ }^{2}$ Gunma Daigaku Daigakuin Igakukei Kenkyuka Igakubu
}

March 20, 2021

\begin{abstract}
Noonan syndrome is a genetic multisystem disorder characterized by distinctive facial features, developmental delay, congenital heart disease, and other conditions. It is associated with mutation of genes encoding the proteins in the RAS-MAPK pathway, including PTPN11. We herein describe the first case of Noonan syndrome complicated with hepatocellular carcinoma.
\end{abstract}

The first reported case of Noonan syndrome complicated with hepatocellular carcinoma.

Satoru Kakizaki ${ }^{1,2}{ }^{*}$, Daisuke Uehara ${ }^{1}$, Hiroki Tojima ${ }^{1}$, Takayoshi Suga ${ }^{1}$, Yuichi Yamazaki ${ }^{1}$, Sato Ken ${ }^{1}$, Norio Kubo ${ }^{3}$, Ken Shirabe ${ }^{3}$, Takayuki Yokota ${ }^{4}$, Kei Shibuya ${ }^{5}$, Tatsurou Maehara ${ }^{6}$, Hideaki Yokoo ${ }^{6}$, Atsushi Naganuma $^{7}$, Toshio Uraoka ${ }^{1}$

${ }^{1}$ Department of Gastroenterology and Hepatology, Gunma University Graduate School of Medicine, Maebashi, Gunma 371-8511, Japan

2 Department of Clinical Research, National Hospital Organization Takasaki General Medical Center, Takasaki, Gunma 370-0829, Japan

${ }^{3}$ Department of General Surgical Science, Gunma University Graduate School of Medicine, Maebashi, Gunma 371-8511, Japan

4 Department of Diagnostic Radiology and Nuclear Medicine, Gunma University Graduate School of Medicine, 3-39-22 Showa, Maebashi, Gunma,

${ }^{5}$ Gunma University Heavy Ion Medical Center, Maebashi, Gunma 371-8511, Japan.,

${ }^{6}$ Department of Human Pathology, Gunma University Graduate School of Medicine, Maebashi, Gunma 371-8511, Japan

7 Department of Gastroenterology, National Hospital Organization Takasaki General Medical Center, Takasaki, Gunma 370-0829, Japan

*Corresponding author

Satoru Kakizaki, M.D., Ph.D., AGAF.

Department of Clinical Research, National Hospital Organization Takasaki General Medical Center, 36 Takamatsu-cho, Takasaki, Gunma 370-0829, Japan. 
Tel: +81-27-322-5108, Fax: +81-27-322-6111, E-mail: kakizaki@gunma-u.ac.jp

Running title: Noonan syndrome with hepatocellular carcinoma.

Word count for the abstract: 49 . Word counts for main text: 1562

\begin{abstract}
Noonan syndrome is a genetic multisystem disorder characterized by distinctive facial features, developmental delay, congenital heart disease, and other conditions. It is associated with mutation of genes encoding the proteins in the RAS-MAPK pathway, including PTPN11. We herein describe the first case of Noonan syndrome complicated with hepatocellular carcinoma.
\end{abstract}

Key words : Noonan syndrome, hepatocellular carcinoma, PTPN11.

\title{
INTRODUCTION
}

Noonan syndrome is a genetic multisystem disorder characterized by distinctive facial features, developmental delay, learning difficulties, short stature, congenital heart disease, renal anomalies, lymphatic malformations, and bleeding difficulties [1-3]. The mutations that cause Noonan syndrome alter genes encoding proteins with roles in the RAS-MAPK pathway, leading to pathway dysregulation [1-3]. Noonan syndrome was first characterized by Jacqueline Noonan, who reported nine patients with pulmonary valve stenosis, small stature, hypertelorism, mild intellectual disability, ptosis, undescended testes, and skeletal malformations [4]. Noonan syndrome is an autosomal dominant, variably expressed, multisystem disorder with an estimated prevalence of 1 in 1,000 to 2,500 [5]. In the RAS-MAPK signaling pathway, molecular genetic testing identifies a pathogenic variant in PTPN11 in 50\% of affected individuals, SOS1 in approximately 13\%, RAF1 and RIT1 in $5 \%$ each, and KRAS in <5\% [1].

Several hematological cancers have been reported in patients with Noonan syndrome, particularly during childhood, including juvenile myelomonocytic leukemia, acute myelogenous leukemia, and B-cell acute lymphoblastic leukemia [6]. A large study of a cohort of 297 Dutch patients with Noonan syndrome and a pathogenic PTPN11 mutation calculated that the risk of cancer was increased 3-5 times in comparison to the general population [7].

It is reported that expression of PTPN11/Shp2 is elevated in human hepatocellular carcinoma (HCC), and that its levels were even higher in metastatic foci [8]. PTPN11 is representative pathogenic gene of Noonan syndrome [1]. PTPN11, which encodes tyrosine phosphatase Shp2, is a critical gene mediating the cellular responses to hormones and cytokines [9]. The loss of Shp2 promotes the development of HCC, suggesting that PTPN11 functions as a tumor suppressor in HCC tumorigenesis [10,11]. As a result, there seems to be a relationship between abnormality of PTPN11 and/or RAS-MAPK signaling pathway in Noonan syndrome and HCC. However, there have been no previous case reports of Noonan syndrome complicated with HCC.

We herein report the case of a patient with Noonan syndrome complicated with HCC. To our knowledge, this represents the first reported case of Noonan syndrome complicated with HCC.

\section{CASE PRESENTATION}

A 19-year-old man consulted our department after hepatic hyperechoic lesions were incidentally pointed out by screening ultrasound. There were no associated symptoms. He had a history of Noonan syndrome. He showed distinctive facial features, as well as developmental delay, mental retardation, funnel chest, hypertrophic cardiomyopathy, mitral regurgitation, and renal anomalies. He had received surgery for an atrial septal defect at 5 years of age, cryptorchidism at 7 years of age, and scoliosis at 19 years of age. His height and weight were $150 \mathrm{~cm}$ and $35 \mathrm{~kg}$, respectively.

The laboratory data at the first visit were as follows: red blood cell count $(\mathrm{RBC}), 314 \times 10^{4} / \mu \mathrm{L}$; hemoglobin (Hb), $14.5 \mathrm{~g} / \mathrm{dL}$; white blood cell count (WBC), 5,500/ $\mu \mathrm{L}$; platelet count (Plt), $13.9 \times 10^{4} / \mu \mathrm{L}$; prothrombin time (PT), $71 \%$; PT-INR, 1.16; total protein (TP), $6.2 \mathrm{~g} / \mathrm{dL}$; albumin (Alb), $3.4 \mathrm{~g} / \mathrm{dL}$; total bilirubin (T-Bil), $2.6 \mathrm{mg} / \mathrm{dL}$; direct bilirubin (D-Bil) $0.1 \mathrm{mg} / \mathrm{dL}$; aspartate aminotransferase (AST) $25 \mathrm{U} / \mathrm{L}$; alanine 
aminotransferase (ALT) $23 \mathrm{U} / \mathrm{L}$; lactate dehydrogenase (LDH) $154 \mathrm{U} / \mathrm{L}$; alkaline phosphatase (ALP), 344 $\mathrm{U} / \mathrm{L} ; \gamma$-glutamyltransferase $(\gamma$-GT), $40 \mathrm{U} / \mathrm{L}$; blood urea nitrogen $(\mathrm{BUN}), 14 \mathrm{mg} / \mathrm{dL}$; creatinine $(\mathrm{Cr}), 0.53$ $\mathrm{mg} / \mathrm{dL}$ and C-reactive protein $(\mathrm{CRP}), 0.01 \mathrm{mg} / \mathrm{dL}$. The patient was negative for both hepatitis B surface antigen and hepatitis C virus antibody. His alpha-fetoprotein (AFP), serum des-gamma-carboxy prothrombin (DCP), carcinoembryonic antigen (CEA), and carbohydrate antigen 19-9 (CA19-9) values were all within the normal ranges.

Abdominal computed tomography $(\mathrm{CT})$ revealed that the portal vein seemed to be interrupted at the liver hilum and flow to the inferior vena cava merging with the hepatic vein (Figure 1a ). The liver parenchyma showed poor contrast and the intrahepatic portal vein was unclear in the portal phase (Figure 1b ). The irregular parenchyma disappeared at the late phase (Figure 1c ). At the lower margin of segment 6, the tumor showed enhancement from the margin, with staining continued until the late phase (Figure 1d, e ). This tumor showed high signal intensity on T2-weighted magnetic resonance imaging (MRI) (Figure 1f ). We considered that the findings reflected a diagnosis of hemangioma. Superparamagnetic iron oxide (SPIO)-enhanced MRI revealed another tumor in segment 4. This tumor showed low signal intensity after SPIO enhancement, while the central portion showed high intensity (Figure 2a ). We considered that this tumor was focal nodular hyperplasia (FNH). Similar lesions were also observed in the liver. We considered that the portal vein and vessel abnormalities associated with Noonan syndrome had caused multiple sites of hyperplasia in the liver. Because it was difficult for the patient to remain still for a long time due to mental retardation, gadolinium-ethoxybenzyl-diethylenetriamine pentaacetic acid (EOB)-enhanced MRI were not performed in the initial evaluation.

The FNH-like lesion in segment 4 gradually grew (Figure 2b, c ). On EOB-MRI, this lesion showed faint staining in the early phase, was unclear in the portal phase and showed central low intensity in the hepatocyte phase (Figure 2d, e, f ). A similar lesion was observed in segment 7 (Figure 3a, b ). On an SPIO-MRI scan performed 2 years after the first visit, the lesion was $16 \mathrm{~mm}$ in diameter. This tumor also gradually grew; however, the growth rate was slow (Figure 3c, d, e ). During the course, hypervascular areas were observed in the tumor of segment 7 in the arterial phase, while washout was observed in the late phase. Although the serum DCP level was within normal range until 3 years from first visit, it was elevated to 116 $\mathrm{mAU} / \mathrm{mL}$ at 4 years from the first visit and then gradually increased. We hypothesized this tumor contained the HCC component.

Surgical resection, transarterial chemoembolization, and radiation therapy were considered. Because of the patient's mental retardation and portal vein abnormality, supportive care was selected. The hepatic tumor gradually grew and reached $12 \times 16 \mathrm{~cm}$ diameter at 7 years from the first visit. Obstructive jaundice developed as a complication of tumor progression. The laboratory data at this time were as follows: $\mathrm{RBC}, 314 \times 10^{4} / \mu \mathrm{L}$;

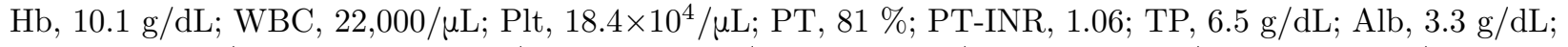
T-Bil, $34.8 \mathrm{mg} / \mathrm{dL}$; D-Bil, $28.1 \mathrm{mg} / \mathrm{dL}$; AST, $82 \mathrm{U} / \mathrm{L} ;$ ALT, $24 \mathrm{U} / \mathrm{L}$; LDH, $199 \mathrm{U} / \mathrm{L} ;$ ALP, $185 \mathrm{U} / \mathrm{L} ; \gamma$-GT, $67 \mathrm{U} / \mathrm{L} ; \mathrm{BUN}, 67 \mathrm{mg} / \mathrm{dL} ; \mathrm{Cr}, 2.27 \mathrm{mg} / \mathrm{dL}$ and CRP, $3.67 \mathrm{mg} / \mathrm{dL}$. AFP was $14.9 \mathrm{ng} / \mathrm{mL}$, and DCP was $42,771 \mathrm{mAU} / \mathrm{mL}$

The patient died of liver failure due to tumor progression in the 7th year after the first visit. Autopsy revealed that liver tumor was HCC. The portal vein was interrupted at the liver hilum and flowed directly into the hepatic vein (Figure 4a ). Microscopic evaluation of the tumor revealed well to moderately differentiated HCC (Figure 4b ). Part of the tumor cells showed foamy cytoplasm and contained fat droplets (Figure 4c ). Histological examination of the background liver showed cholestasis, fibrosis around the portal vein and central vein, and ductular proliferation (Figure 4d ).

\section{DISCUSSION}

We reported the first case of Noonan syndrome complicated with HCC. Noonan syndrome is a genetic multisystem disorder that involves mutations of genes encoding the proteins in the RAS-MAPK pathway [1-3]. PTPN11 is representative pathogenic gene of Noonan syndrome [1]. PTPN11, which encodes tyrosine phosphatase Shp2, is a critical gene mediating cellular responses to hormones and cytokines [10]. Activating 
mutations in PTPN11 have been shown to be directly associated with the pathogenesis of Noonan syndrome and childhood leukemias $[6,13]$. In Noonan syndrome patients with a pathogenic PTPN11 mutation, the risk of developing cancer is reported to be 3-5 times higher than that in the general population [7]. The loss of Shp2 promotes the development of HCC, suggesting that PTPN11 functions as a tumor suppressor in HCC tumorigenesis [10, 11]. PTPN11 was first identified as a proto-oncogene in leukemia [13]. However, most recent findings suggest that PTPN11 plays a tumor suppressor role in HCC $[10,11]$, implying its dual faces in tumorigenesis.

Although the defined pathogenesis of HCC in the present case was not confirmed, we considered that there were two possibilities. One possibility involves genetic abnormalities associated with Noonan syndrome. Because Noonan syndrome is associated with mutations in PTPN11 and/or genes encoding the RAS-MAPK pathway [1-3], these pathogenic variants may have caused HCC in the present case. PTPN11, which is mutated in Noonan syndrome, has tumor suppressor roles in HCC [10, 11]. Patients with Noonan syndrome seem to have an increased risk of developing HCC.

The other possible pathogenesis involves vessel abnormality and congestion. Chronic congestion due to heart failure is one of the pathogeneses of HCC [13]. The present case was complicated by hypertrophic cardiomyopathy, and mitral regurgitation. Furthermore, the patient had past history of surgery for an atrial septal defect. The patient also had a portal vein anomaly. It is known that increased venous pressure and decreased cardiac output and hepatic venous drainage result in sinusoidal dilatation around the central veins [13]. Finally, this may have caused liver cirrhosis and the development of HCC. Chronic congestion and/or vessel abnormality may be a reason for the development of HCC in this case.

One limitation of this case report is that gene mutation analyses of PTPN11 and/or RAS-MAPK pathway proteins were not performed. Because this is the only case report on Noonan syndrome complicated with HCC, it would be desirable to accumulate and analyze other cases. Although this is the only reported case of Noonan syndrome complicated with HCC and despite the relationship between these two diseases being unknown, the presence of RAS-MAPK pathway protein abnormalities and the development of HCC are clinically interesting.

\section{Conflict of Interest}

The authors declare no conflicts of interest in association with the report.

\section{References}

1. Allanson, JE, Roberts AE. Noonan syndrome. Retrieved from https://www.ncbi.nlm.nih.gov/books/NBK1124/ 2019, August 8.

2. Roberts AE, Allanson JE, Tartaglia M, Gelb BD. Noonan syndrome.Lancet. 2013; 381: 333-42.

3. Romano AA, Allanson JE, Dahlgren J, et al. Noonan syndrome: clinical features, diagnosis, and management guidelines. Pediatrics.2010; 126: 746-59.

4. Noonan JA, Ehmke DA. Associated noncardiac malformations in children with congenital heart disease. Midwest Soc Pediatr Res. 1963; 63: 468-70.

5. Mendez HM, Opitz JM. Noonan syndrome: a review. Am J Med Genet.1985; 21: 493-506.

6. Tartaglia M, Gelb BD. Germ-line and somatic PTPN11 mutations in human disease. Eur J Med Genet. 2005; 48: 81-96.

7. Jongmans MC, van der Burgt I, Hoogerbrugge PM, et al. Cancer risk in patients with Noonan syndrome carrying a PTPN11 mutation. Eur J Hum Genet. 2011; 19: 870-4.

8. Han T, Xiang DM, Sun W, et al. PTPN11/Shp2 overexpression enhances liver cancer progression and predicts poor prognosis of patients.J Hepatol. 2015; 63: 651-60.

9. Bard-Chapeau EA, Li S, Ding J, et al. Ptpn11/Shp2 acts as a tumor suppressor in hepatocellular carcinogenesis. Cancer Cell. 2011; 19: 629-39.

10. Jiang C, Hu F, Tai Y, et al. The tumor suppressor role of Src homology phosphotyrosine phosphatase 2 in hepatocellular carcinoma. J Cancer Res Clin Oncol. 2012; 138:637-46. 
11. Grossmann KS, Rosário M, Birchmeier C, et al. The tyrosine phosphatase Shp2 in development and cancer. Adv Cancer Res. 2010; 106: 53-89.

12. Loh ML, Vattikuti S, Schubbert S, et al. Mutations in PTPN11 implicate the SHP-2 phosphatase in leukemogenesis. Blood. 2004; 103: 2325-31.

13. Kogiso T, Tokushige K. Fontan-associated liver disease and hepatocellular carcinoma in adults. Sci Rep. 2020; 10: 21742.

Figure 1. The computed tomography $(\mathrm{CT})$ and magnetic resonance imaging (MRI) findings.a : The portal vein seemed to be interrupted at the liver hilum and flow into inferior vena cava, merging with the hepatic vein (arrow).b : The liver parenchyma showed poor contrast and the intrahepatic portal vein was unclear in the portal phase, $\mathbf{c}$ : The irregular parenchyma disappeared in the late phase. $\mathbf{d}, \mathbf{e}:$ At the lower margin of segment 6, the tumor (arrow) showed enhancement from the margin (d) and staining continued until the late phase (e).f : The tumor showed high signal intensity on T2-weighted MRI.

Figure 2. The magnetic resonance imaging (MRI) findings.a, b, c: Superparamagnetic iron oxide (SPIO)enhanced MRI. A tumor was detected in segment 4. This tumor showed low signal intensity after SPIO enhancement and the central portion showed high signal intensity. (a ), (b ): One year after the first visit. (c) : Two years after the first visit. $\mathbf{d}, \mathbf{e}, \mathbf{f}:$ gadolinium-ethoxybenzyl-diethylenetriamine pentaacetic acid (EOB)-enhanced MRI at 3.5 years after the first visit. The tumor showed faint staining in the early phase (d), unclear in the portal phase (e ) and central low intensity in the hepatocyte phase (f ).

Figure 3. The magnetic resonance imaging (MRI) findings of the tumor located in segment 7 . a , $\mathbf{b}$ : Three years after the first visit. The tumor showed faint staining in the early phase (a), and low to iso intensity in the hepatocyte phase (b ). c, d, e : This tumor gradually grew, although the growth rate was slow. During the course, hypervascular areas were observed in the arterial phase and washout was observed in the late phase. (c) : Four years after the first visit. (d) : Five years after the first visit. (e) : Six years after the first visit.

Figure 4. Autopsy findings. a: The portal vein was interrupted at the liver hilum and flowed directly into the hepatic vein. $\mathbf{b}$ : Microscopic findings of the tumor lesion demonstrated well to moderately differentiated HCC. $\mathbf{c}$ : Part of the tumor cells showed foamy cytoplasm and contained fat droplets. $\mathbf{d}$ : The histology of the background liver showed cholestasis, fibrosis around portal vein and central vein, and ductular proliferation. H.E., hematoxylin and eosin staining.

\section{Hosted file}

Noonan.Figure.pptx available at https://authorea.com/users/402786/articles/514548-the-firstreported-case-of-noonan-syndrome-complicated-with-hepatocellular-carcinoma 